\title{
FALLA CARDÍACA DESCOMPENSADA: ESTRATIFICACIÓN DEL RIESGO DE MORTALIDAD INTRAHOSPITALARIA HOSPITAL DE SAN JOSÉ DE BOGOTÁ DC.
}

Cristina Henao MD*, Javier Mauricio Benavides B. MD*, Walter Chaves MD**, Juan José Diaztagle MD***, John Sprokel MD***, José Ignacio Hernández MD****

\section{Resumen}

Introducción: la falla cardíaca es una de las principales causas de consulta en urgencias y de mortalidad intrahospitalaria.Objetivo: describir el riesgo de esta última mediante dos instrumentos, a quienes ingresaron con falla cardíaca descompensada al servicio de medicina interna del Hospital de San José de Bogotá DC. Métodos: se reclutaron 155 pacientes entre marzo de 2010 y febrero de 2011, aplicando el score de riesgo GWTG-HF y el nomograma de predicción de riesgo del estudio OPTIMIZE, describiendo variables clínicas y demográficas. Se reporta la mortalidad esperada y la observada. Resultados: la edad promedio fue 72.5 (DE 12.1) años. Se documentó muerte intrahospitalaria en 7,7\% (n 12). Para la estratificación de riesgo se incluyeron 146 casos. El 64,3\% se clasificó en riesgo de 1 a $5 \%$ por la herramienta de la AHA y 50,7\% utilizando el nomograma OPTIMIZE HF. Más de la mitad de los fallecidos tenían bajo riesgo (1\% a $5 \%$ ). No se encontró concordancia entre los métodos ( $\operatorname{Lin} 0.07$ ). Entre los fallecidos los niveles de pro BNP mayores de $12.500 \mathrm{pg} / \mathrm{ml}$ y la arritmia al ingreso fueron reportados con frecuencia. Estas variables no son tenidas en cuenta por los instrumentos utilizados. Conclusión: el uso de índices de riesgo para muerte intrahospitalaria puede ser una herramienta útil, pero se requieren otros estudios para aplicarlos en nuestra población. Palabras clave: insuficiencia cardíaca, mortalidad, factores de riesgo.

Abreviaturas: FC, falla cardíaca; MIH, mortalidad intrahospitalaria.

\section{RISK STRATIFICATION FOR IN-HOSPITAL MORTALITY IN ACUTELY DECOMPENSATED HEART FAILURE}

\begin{abstract}
Introduction: heart failure is one of the main reasons for consultation to the emergency room and of in-hospital mortality. Objective: to describe the risk of the latter by means of applying two instruments to patients who were hospitalized with acute decompensated heart failure at the internal medicine service at Hospital de San José de Bogotá DC. Methods: 155 patients were recruited between March 2010 and February 2011, applying the GWTG-HF risk score and the OPTIMIZE Study Risk-Prediction Nomogram, describing clinical and demographic variables. The expected and observed mortality is reported. Results: patients had an average age of 72.5 (SD 12.1) years. In-hospital mortality was documented in $7.7 \%$ (n 12). Risk stratification was performed in 146 cases. $64.3 \%$ were classified within risk 1 to $5 \%$ by means of the AHA tool and $50.7 \%$ using the OPTIMIZE HF Nomogram. More than half of the demised patients had a low risk (1\% to 15\%). No concordance between methods was found (Lin 0.07). Pro BNP levels greater than 12.500 $\mathrm{pg} / \mathrm{ml}$ and arrhythmia at the moment of admission were frequently reported among the group of deceased patients. These variables are not considered by the instruments that were used. Conclusions: the use of risk rates for in-hospital mortality may be a useful tool. Nevertheless, other studies are required in order to apply them on our population.
\end{abstract}

Key words: heart failure, mortality, risk factors.

Fecha recibido: junio 16 de $201 \mathrm{I}$ - Fecha aceptado: agosto 8 de $201 \mathrm{I}$

* Residente III de Medicina Interna, Fundación Universitaria de Ciencias de la Salud, Bogotá DC.

** Instructor Asociado, Fundación Universitaria de Ciencias de la Salud.Jefe del Servicio de Medicina Interna, Hospital de San José. Bogotá DC.
*** Instructor Asistente, Fundación Universitaria de Ciencias de la Salud. Bogotá DC.

* Especialista en Medicina Interna y Cardiología. Profesor Emérito, Fundación Universitaria de Ciencias de la Salud. Bogotá DC. 


\section{Introducción}

La falla cardíaca es una de las enfermedades más prevalentes en la población adulta. Datos de Estados Unidos estiman una incidencia de 10 por 1.000 habitantes mayores de 65 años. ${ }^{1}$ En Colombia las estadísticas acerca de prevalencia son escasas; sin embargo, la tasa de mortalidad por FC es de 9.3 por 100.000 habitantes. ${ }^{2}$ La FC descompensada es una causa frecuente de consulta en urgencias y hospitalización en los servicios de medicina interna.

Se ha asociado con una alta tasa de mortalidad tanto intrahospitalaria como en los meses posteriores al alta. En el estudio Framingham el 50\% de los pacientes diagnosticados con FC murieron dentro de los siguientes cinco años posteriores al diagnóstico. ${ }^{3} \mathrm{La}$ MIH varía desde el 2,3\% en pacientes reclutados en ensayos clínicos, hasta $19 \%$ en series de hospitales de referencia. ${ }^{4}$ En nuestra institución se realizó un estudio retrospectivo durante el 2007 y 2008, en el cual se analizaron 129 pacientes con FC, que reportó MIH de $4,2 \% .{ }^{5}$ La FC descompensada se encuentra entra las primeras seis causas de hospitalización por el servicio de medicina interna.

Se han descrito diversas variables asociadas con mortalidad, entre ellas están: edad, frecuencia cardíaca, tensión arterial, concentración de sodio sérico, nitrógeno ureico, creatinina, fracción de eyección del ventrículo izquierdo y clase funcional ${ }^{6-10}$, que han generado modelos predictivos de riesgo con el objeto de mejorar el enfoque terapéutico de aquellos factores que influyen en la MIH. Se han propuesto diferentes herramientas de medición de riesgo como la escala GWTG-HF de la American Heart Association $(A H A)^{11}$, el modelo de predicción de sobrevida de Seattle ${ }^{12}$ y el nomograma de predicción de riesgo ${ }^{8}$; sin embargo, estas herramientas no suelen utilizarse en nuestro medio.

El objetivo de este análisis es evaluar el riesgo de MIH utilizando la escala GWTG-HF y el nomograma de predicción de riesgo del estudio OPTIMIZE, en pacientes que ingresan con diagnóstico de FC descompensada al servicio de medicina interna del Hospital de San José de Bogotá DC. El objetivo secundario es describir la concordancia entre las escalas.

\section{Métodos}

Diseño: a todos los pacientes reclutados entre marzo de 2010 y febrero de 2011 se les aplicó la escala de riesgo GWTG-HF y el nomograma de predicción de riesgo del estudio OPTIMIZE. Se incluyeron pacientes mayores de 18 años hospitalizados en el servicio de medicina interna con diagnóstico de FC descompensada según los criterios de Framingham. El diagnóstico se realizó con al menos un criterio mayor y dos menores. Son criterios mayores: disnea (paroxística nocturna, ortopnea), ingurgitación yugular, estertores, cardiomegalia (aumento del índice cardiotorácico mayor de 0.5 en la placa de tórax PA), edema pulmonar, galope por tercer ruido, presión venosa central mayor de $16 \mathrm{~cm}$ de $\mathrm{H}_{2} \mathrm{O}$ y reflujo hepatoyugular. Son criterios menores: tos nocturna, derrame pleural, edema bilateral de miembros inferiores, disnea de esfuerzo, hepatomegalia, taquicardia (frecuencia cardíaca > 120 latidos/minuto) y pérdida de más de $4.5 \mathrm{~kg}$ de peso tras cinco días de tratamiento.

Se excluyeron los pacientes con concurrencia de descompensación aguda de diabetes mellitus, urgencia dialítica, cirrosis hepática CHILD C/C o insuficiencia hepática aguda, síndrome nefrótico, choque hipovolémico o séptico de cualquier origen y patología neoplásica terminal.

Para el estudio se describieron las variables demográficas y las clínicas: clase funcional de base (últimos 3 meses), clase funcional de ingreso según clasificación de New York Heart Association, ${ }^{13}$ comorbilidades, antecedentes farmacológicos (medicamentos administrados en el último mes previo al ingreso) y signos vitales.

$\mathrm{Al}$ ingreso se hicieron mediciones de nitrógeno ureico, creatinina y sodio séricos, y hemoglobina, procesadas con técnica de química seca VITROS 250. Se definió hiponatremia como sodio menor de $135 \mathrm{mEg} / \mathrm{l}$ y anemia con hemoglobina menor o igual a $10.7 \mathrm{~g} / \mathrm{dl}$ en 
mujeres y $11.7 \mathrm{~g} / \mathrm{dl}$ en hombres (nivel de hemoglobina ajustada a 2.600 metros de altura según criterios de la OMS). ${ }^{14} \mathrm{El}$ propéptido natriurético tipo B (pro BNP) y la troponina I se midieron dentro de las primeras 24 horas de reclutamiento y fueron procesadas en VITROS 250 con técnica de quimioluminiscencia inmunométrica.

A todos los pacientes se les realizó electrocardiograma de doce derivaciones y ecocardiograma transtorácico con equipo SONOX 5500, excepto cuando en la historia del paciente se documentara reporte ecocardiográfico los tres meses previos.

Se aplicó la escala GWRG-HF de la American Heart Association (AHA), ${ }^{11}$ que propone siete variables: edad, tensión arterial sistólica, nitrógeno ureico y sodio séricos, frecuencia cardíaca, antecedente de EPOC y raza negra; la sumatoria de puntajes otorgados a cada variable establece un puntaje en el rango de 0 a 100 , el cual se extrapola a la probabilidad de MIH. Se determina una relación directamente proporcional entre puntaje y probabilidad de muerte, es decir, a mayor puntaje mayor probabilidad de MIH. La herramienta se encuentra disponible en (http://www.americanheart. org/presenter.jhtml ?identifier=3027533). Se compararon los resultados con el monograma de predicción de riesgo ${ }^{8}$, generando de un modelo multivariado realizado con la población del registro OPTIMIZE-HF basado en siete variables: edad, frecuencia cardíaca, tensión arterial sistólica, sodio sérico, creatinina, causa primaria del ingreso y presencia de disfunción sistólica del ventrículo izquierdo, definida como fracción de eyección menor de $40 \%$, se asigna a cada variable descrita un puntaje y la sumatoria de éstos se introduce al nomograma disponible en www.optimize-hf.org estadificando la probabilidad de muerte de cada paciente.

Análisis estadístico: se realizó uno descriptivo de las variables cuantitativas a través de medidas de tendencia central y dispersión; las variables cualitativas se describen por medio de proporciones. El análisis de los datos se realizó con el programa STATA 10巴. La concordancia entre los métodos se evaluó utilizando el índice de correlación concordancia de Lin.

El porcentaje de datos no registrados se reporta a continuación: sodio sérico en cuatro $(2,6 \%)$, nitrógeno ureico en tres $(1,9 \%)$, creatinina sérica en uno $(0,6 \%)$ y la fracción de eyección en cinco $(3,2 \%)$ pacientes. Solo se tuvieron en cuenta para este análisis aquellos con registro completo de las variables evaluadas con las dos herramientas de medición de riesgo. El estudio fue aprobado por el comité de investigación y ética de la Facultad de Medicina de la Fundación Universitaria de Ciencias de la Salud, Hospital de San José de Bogotá DC.

\section{Resultados}

Se tuvieron en cuenta para análisis de los parámetros basales 155 pacientes y se aplicó el puntaje de las escalas a 146 (Figura 1). Las características de los pacientes se presentan en la Tabla 1. El promedio de edad fue 72.5 (DE 12.1) años, con mayor proporción entre 70 y 89 años. La razón hombre/mujer fue 1.1: 1 . Al ingreso el $60 \%$ se encontraba en clase funcional NYHA IV. Las comorbilidades identificadas con mayor frecuencia fueron hipertensión arterial, EPOC y diabetes, $78 \%, 48 \%$ y $25 \%$. El $54,2 \%$ refería antecedente de falla cardíaca y el $18,7 \%$ reportó antecedente de enfermedad coronaria. En cuanto a los laboratorios de ingreso, 38,3\% tenía creatinina sérica mayor o igual a $1.2 \mathrm{mg} / \mathrm{dl}$. La prevalencia de anemia fue de $20 \%$ y la proporción de pacientes con hiponatremia $27,7 \%$. Los marcadores de disfunción miocárdica mostraron troponina I positiva en $27,3 \%$ y el 96\% registró pro-BNP en rango diagnóstico para falla cardíaca. La fibrilación auricular fue la arritmia más frecuente, identificada en el 25,8\%. La fracción de eyección se encontró por debajo de $40 \%$ en $35,3 \%$ de los pacientes. 


\section{Tablal. Características basales de la población}

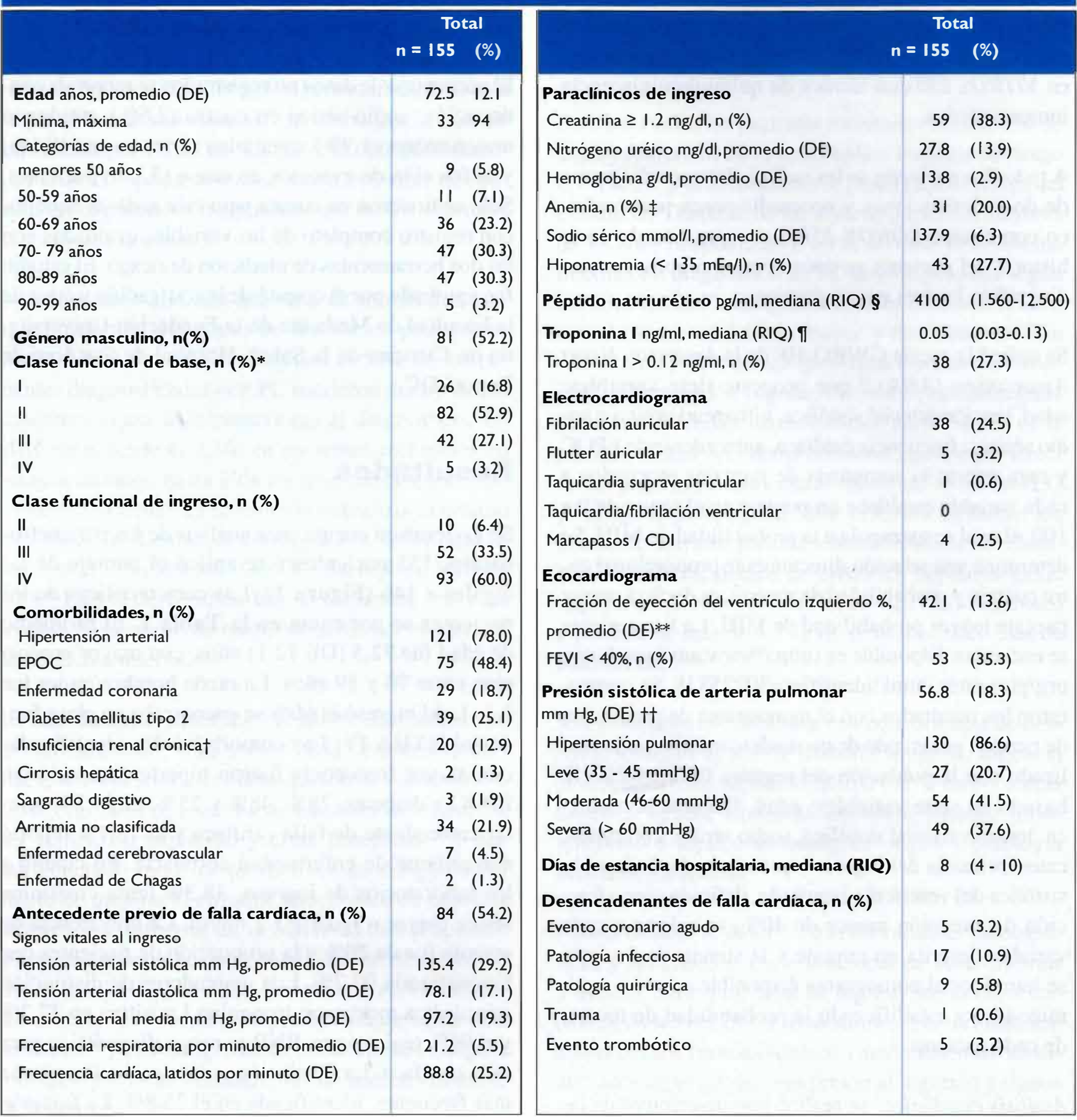

* Clase funcional referida en los tres meses previos al ingreso, clasificación NYHA. † Insuficiencia renal crónica clasificación KDOQI $\mathrm{TFG} \leq 60 \mathrm{ml} / \mathrm{mn}$. $\ddagger$ Anemia definida según OMS para hombres $<11,7 \mathrm{~g} / \mathrm{dl}$, para mujeres $<10,7 \mathrm{~g} / \mathrm{dl}$. § Datos disponibles para 141 pacientes. II Datos disponibles para 139 pacientes. ** Datos disponibles para 150 pacientes. †† Hipertensión pulmonar definida como

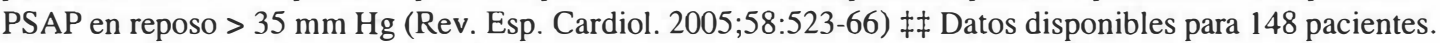




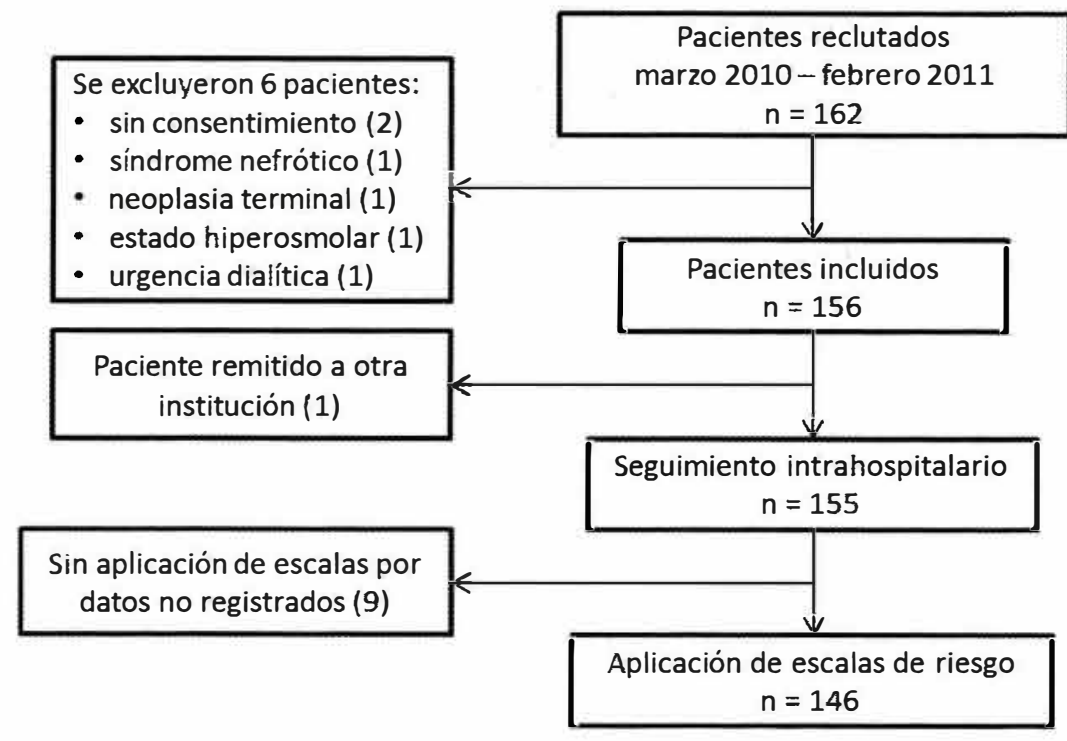

Figura I. Flujograma de reclutamiento y seguimiento de pacientes.

Al realizar la estratificación de riesgo de muerte intrahospitalaria se encontró riesgo de uno a $10 \%$ en el $69 \%$ por criterios AHA, GWTG-HF y $63 \%$ utilizando el nomograma OPTIMIZE HF. Ningún instrumento registró riesgo superior al 30\% (Tabla 2). En nuestra serie la mayoría de fallecidos tenía puntaje de riesgo de muerte entre el 5\% y 10\%. La Figura 2 representa los límites de concordancia de Blant y Altman para los instrumentos de medición de riesgo. No se encontró concordancia entre los métodos (Lin 0.07). El promedio de las diferencias entre los dos puntajes fue 5,9\% con límites de confianza (-17.9 - 29.7).

Se documentó muerte intrahospitalaria en 7,7\% (n 12). Entre los fallecidos una tercera parte ingresó por causas diferentes a falla cardíaca, la mitad tenía rango de edad entre 70-79 años. Se registró taquicardia en tres cuartas partes de esta población. Dos terceras partes se presentaron con elevación de azoados (creatinina $>1,2$ $\mathrm{mg} / \mathrm{ml}$ ), al ingreso la mitad de los pacientes presentó niveles de pro BNP mayores a $12.500 \mathrm{pg} / \mathrm{ml}$, con troponina I positiva en una tercera parte de pacientes, sin documentar en ellos la presencia de infarto agudo de miocardio. Más de la mitad de los fallecidos registró arritmia al ingreso (Tabla 2).
Por criterios AHA GWTG-HF, tres cuartas partes de los pacientes fallecidos habían sido clasificados con riesgo de $\mathrm{MIH}$ del $1 \%$ al 5\%. En contraste, en la estratificación de riesgo con el nomograma OPTIMIZE la mitad de los fallecidos habían sido clasificados con riesgo entre $1 \%$ y $5 \%$ (Tabla 3 ).

\section{Discusión}

En diferentes estudios se han identificado numerosas variables que se asocian con aumento de la MIH en falla cardíaca descompensada, permitiendo el desarrollo de modelos para la estratificación de riesgo. ${ }^{7.15} \mathrm{Sin}$ embargo, a pesar del aumento dramático de la carga a los sistemas de salud, el uso de estas herramientas durante la admisión a urgencias no está bien establecido. ${ }^{8,16}$

Aunque, se ha documentado que la MIH varía desde $3,8 \%$ hasta $23 \%, 6,8,11$ al realizar el cálculo con dos herramientas diferentes encontramos que el riesgo de esta eventualidad fue menor de $10 \%$ para la mayoría de nuestra población. Sin embargo, la mortalidad observada fue elevada $(7,7 \%)$, como se observa en el estudio de García y cols. de la Fundación Cardiovas- 


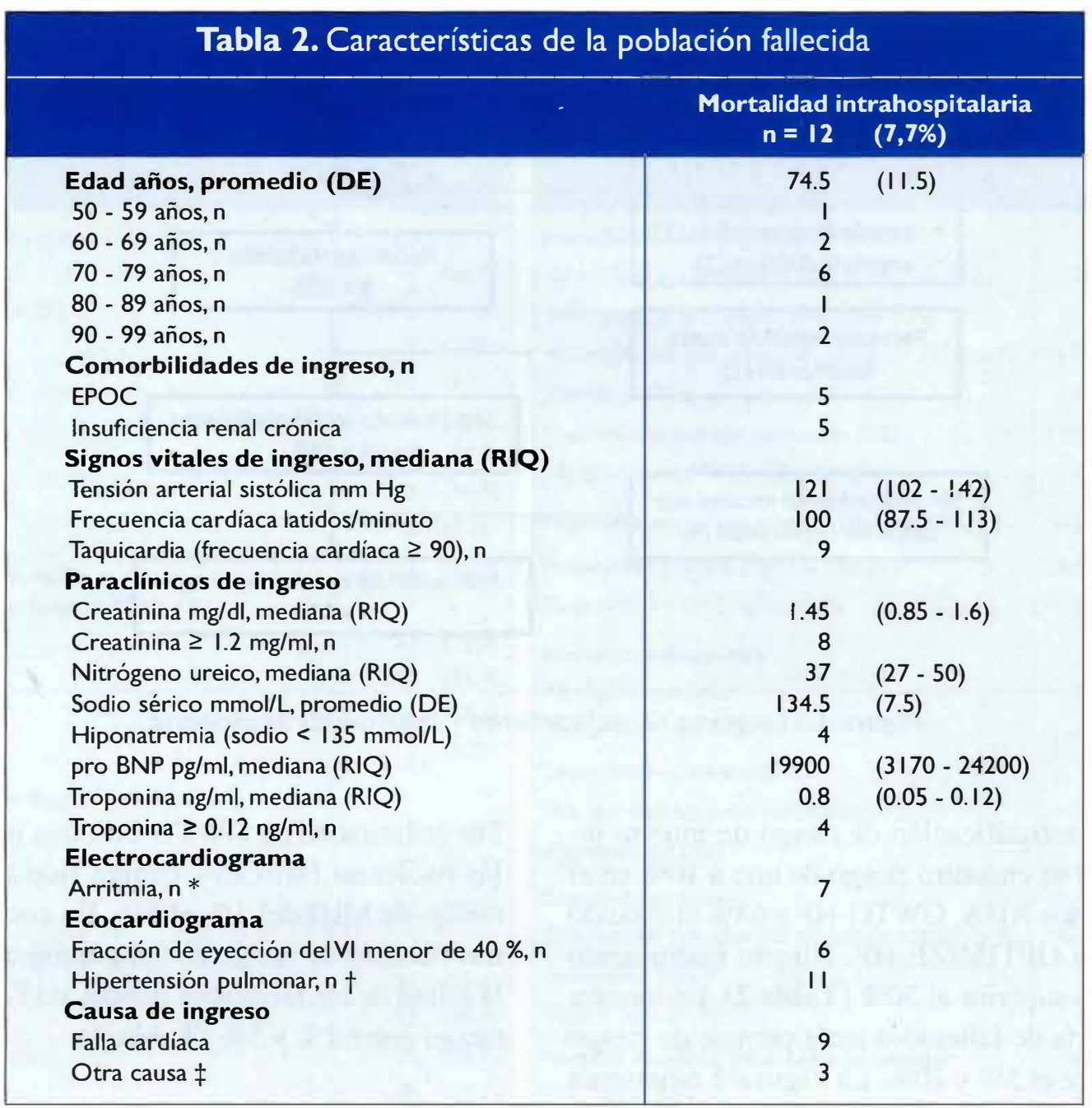

* Incluye fibrilación auricular, flutter auricular y taquicardia supraventricular. $†$ Definida como PSAP en reposo > 35 mm Hg (Rev. Esp. Cardiol. 2005;58:523-66). ‡ Otras causas de ingreso: un paciente por colecistitis y dos por enfermedad cerebrovascular.

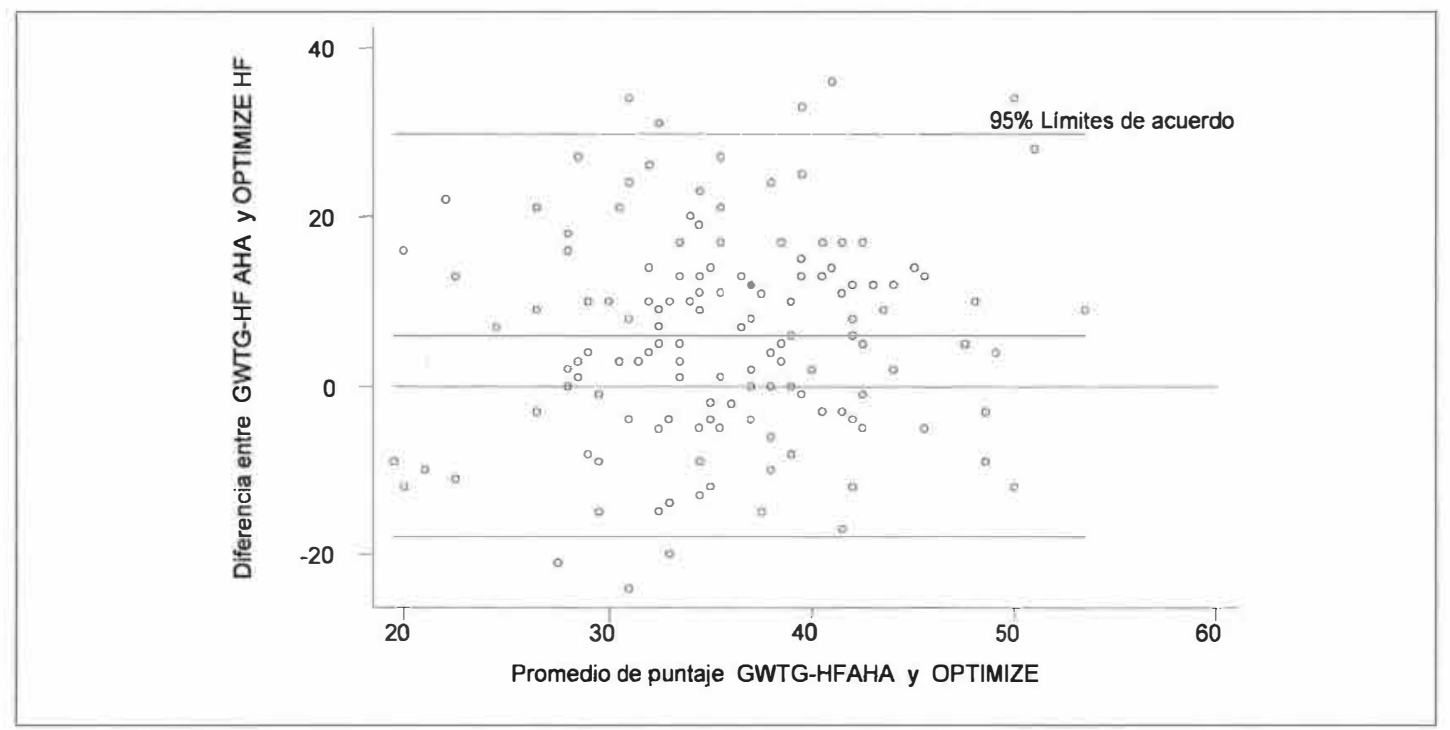

Figura 2. Límites de concordancia de Blant y Altman entre el puntaje GWTG-HFAHA y OPTIMIZE. 
cular del Oriente Colombiano en Bucaramanga, que reportó $9,3 \%^{17}$, sugiriendo que se deben considerar variables diferentes a los predictores evaluados. Se sugiere valorar la asociación de marcadores de disfunción miocárdica y la adherencia a protocolos y guías de manejo aplicados en los centros de atención hospitalaria, pero esto sobrepasa los alcances de nuestro estudio.

Al comparar la población reclutada con estudios de mayor impacto demográfico como ADHERE y OPTIMIZE, encontramos que el promedio de edad es similar, pero la incidencia de comorbilidades como insuficiencia renal crónica (12\%) y enfermedad coronaria para nuestra población es baja (18\%), comparadas con el registro ADHERE (59\%) y el OPTIMIZE (46\%), mientras la prevalencia de EPOC (48\%) y cor pulmonar $^{18}$ en los pacientes reclutados fueron más elevadas.

Dentro de los marcadores de riesgo descritos, el deterioro de la función renal fue un hallazgo frecuente, pero, al comparar con el registro ADHERE y OPTIMIZE se debe tener en cuenta que el promedio de creatinina para estas series fue más alto (cerca de 1.8 a $2.1 \mathrm{mg} / \mathrm{ml}$ ), comparado con nuestra población (1.1 $\mathrm{mg} / \mathrm{ml}$ ). En cuanto a los marcadores de disfunción miocárdica, la troponina I mayor de $0.12 \mathrm{ng} / \mathrm{ml}$ y pro BNP mayor a $12.500 \mathrm{pg} / \mathrm{ml}$, así como la presencia de arritmia cardíaca, fueron prevalentes en el grupo de fallecidos de nuestro estudio, destacando que estos marcadores no se incluyen en las herramientas de riesgo utilizadas, lo cual podría en cierta medida cambiar los grupos de riesgo al introducir estas variables a dichos instrumentos.

Se han propuesto diversas herramientas para estratificar el riesgo de muerte hospitalaria, aunque nosotros no encontramos concordancia entre las dos herramientas utilizadas. A diferencia del nomograma para el cálculo del riesgo, la escala propuesta por la AHA es práctica y permite la medición de variables clínicas a la cabecera del enfermo; sin embargo, consideramos que debe validarse para aplicarlas en nuestra población.

Dentro de las fortalezas de este estudio resaltamos que en Colombia existen pocas investigaciones para la caracterización demográfica de la población con falla cardíaca y la MIH asociada con su descompensación y no existen reportes en el país que analicen el riesgo de muerte utilizando alguna de las herramientas descritas. Los resultados obtenidos permiten la identificación de potenciales variables que podrían influir en la $\mathrm{MIH}$ de pacientes con FC, tales como la troponina I y el $\mathrm{BNP}$, a pesar de que no fueron tenidas en cuenta en las herramientas empleadas para estratificación de riesgo. No se evaluaron otras variables como reingreso a la institución, tabaquismo, índice de masa corporal, ni antecedente de dislipidemia. El objeto de este estudio no fue validar los instrumentos de estratificación de riesgo sino aplicarlos en la población de estudio.

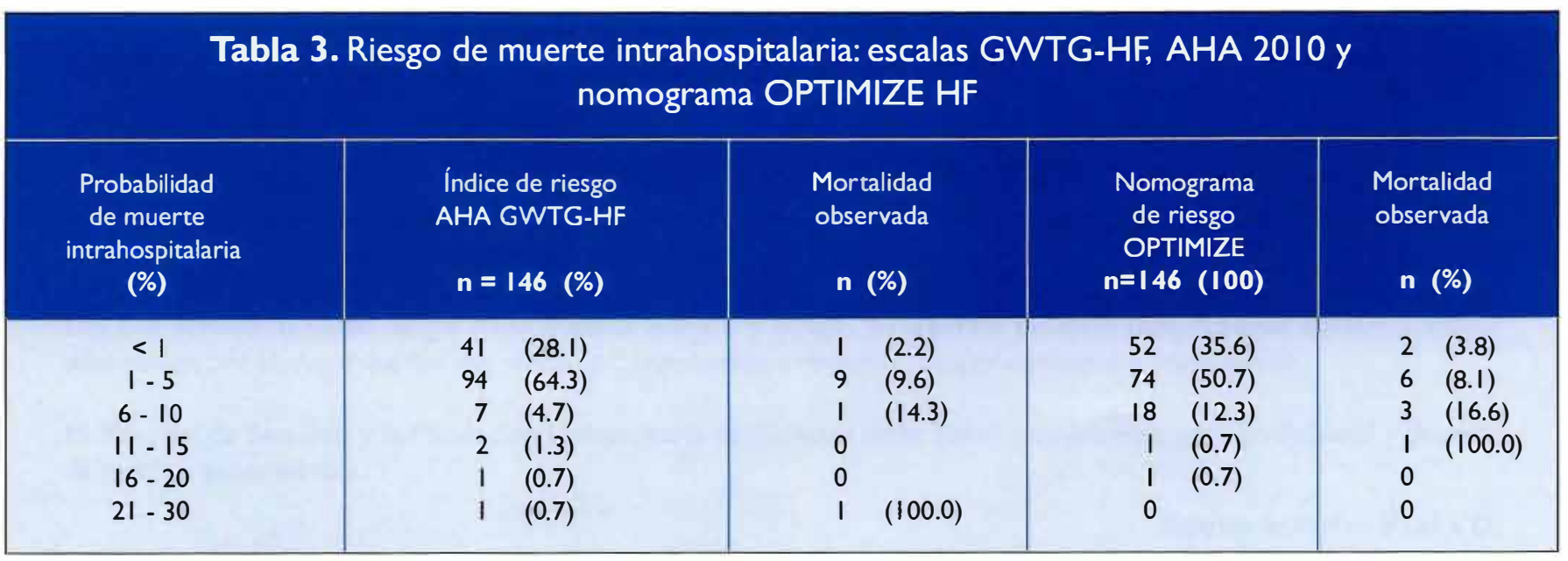




\section{Conclusión}

El uso de índices predictores de riesgo para MIH puede ser una herramienta útil para el enfoque del paciente con FC que ingresa al servicio de medicina interna, pero consideramos que se requieren otros estudios para validarlos de acuerdo con nuestra población y evaluar factores de riesgo adicionales.

\section{Referencias}

1. Lloyd-Jones D, Adams R, Camethon M, et al. Heart disease and stroke statistics--2009 update: a report from the American Heart Association Statistics Committee and Stroke Statistics Subcommittee. Circulation. 2009;119(3):480-86.

2. OMS. Enfermedades cardiovasculares [monografía en Internet]. Washington: OMS; 2011 [citado 30 Sep. 2011]. Disponible en: http://www.who.int/mediacentre/factsheets/fs $317 /$ es/index.html

3. McKee PA, Castelli WP, McNamara PM, Kannel WB. The natural history of congestive heart failure: the Framingham study. N Engl J Med. 1971; 285(26): 1441-46.

4. Nante N, De Marco MF, Balzi D, Addari P, Buiatti E. Prediction of mortality for congestive heart failure patients: results from different wards of an Italian teaching hospital. Eur J Epidemiol. 2000;16(11):1017-1021.

5. Lancheros A, Valencia Y, Chaves Santiago W. Insuficiencia cardiaca aguda: Factores asociados con mortalidad. Reper. med. cir. 2009;18(3):166-74.

6. Chen MC, Chang HW, Cheng CI, Chen YH, Chai HT. Risk stratification of inhospital mortality in patients hospitalized for chronic congestive heart failure secondary to non-ischemic cardiomyopathy. Cardiology. 2003; 100(3):136-42.

7. Hunt SA, Baker DW, Chin MH et al. ACC/AHA guidelines for the evaluation and management of chronic heart failure in the adult: executive summary. J Heart Lung Transplant. 2002; 21(2):189-203.
8. Abraham WT, Fonarow GC, Albert NM et al. Predictors of in-hospital mortality in patients hospitalized for heart failure: insights from the Organized Program to Initiate Lifesaving Treatment in Hospitalized Patients with Heart Failure (OPTIMIZE-HF). J Am Coll Cardiol. 2008;52(5):347-56.

9. Polanczyk CA, Rohde LE, Philbin EA, Di Salvo TG. A new casemix adjustment index for hospital mortality among patients with congestive heart failure. Med Care. 1998; 36(10):1489-99.

10. Lee DS, Austin PC, Rouleau JL, Liu PP, Naimark D, Tu JV. Predicting mortality among patients hospitalized for heart failure: derivation and validation of a clinical model. JAMA 2003; 19;290(19):2581-87.

11. Peterson PN, Rumsfeld JS, Liang L, et al. A validated risk score for in-hospital mortality in patients with heart failure from the American Heart Association get with the guidelines program. Circ Cardiovasc Qual Outcomes. 2010; 3(1):25-32.

12. Levy WC, Mozaffarian D, Linker DT et al. The Seattle Heart Failure Model: prediction of survival in heart failure. Circulation. 2006; 113(11):1424-1433.

13. The Criteria Committee of the New York Heart Association. In: Dolguin M. Nomenclature and criteria for diagnosis of diseases of the heart and great vessels. 9th ed. Boston, Mass: Little Brown; 1994. p. 253-56.

14. Redondo-Bermejo B, Pascual-Figal DA, Hurtado-Martinez JA, et al. [Clinical determinants and prognostic value of hemoglobin in hospitalized patients with systolic heart failure]. Rev Esp Cardiol. 2007; 60(6):597-606

15. Fonarow GC, Adams KF, Jr., Abraham WT, Yancy CW, Boscardin WJ. Risk stratification for in-hospital mortality in acutely decompensated heart failure: classification and regression tree analysis. JAMA. 2005; 293(5):572-580.

16. Kerzner R, Gage BF, Freedland KE, Rich MW. Predictors of mortality in younger and older patients with heart failure and preserved or reduced left ventricular ejection fraction. Am Heart J. 2003; 146(2):286-290.

17. García R, Casas J, Silva F, Cubillos L. Factores predictores de mortalidad en pacientes con insuficiencia cardiaca congestiva. 2002. Trabajo No publicado

18. MacNee W. Pathophysiology of cor pulmonale in chronic obstructive pulmonary disease. Part One. Am J Respir Crit Care Med. 1994; 150(3):833-852. 